\title{
MEMORIA COLECTIVA Y BIOGRAFÍAS EDUCATIVAS DE ALFABETIZADORES EN MOVIMIENTOS SOCIALES
}

\author{
SANDRA MABEL LLOSA \\ https://orcid.org/0000-0001-5384-1135 \\ Universidad de Buenos Aires
}

RESUMEN Este artículo se dirige a compartir aspectos metodológicos de una línea de indagación en curso, enfocándose en los desafios de un abordaje colectivo y participativo para la reconstrucción de la memoria colectiva y de las biografias educativas de alfabetizadores de personas jóvenes y adultas en organizaciones y movimientos sociales de Argentina. Se presenta, en primer lugar, la investigación marco en la cual se inscribe este trabajo. Dicha investigación se aboca, a través de una estrategia metodológica general combinada cualitativa y participativa, al estudio de los procesos pedagógicos y psicosociales que dan cuenta de la construcción colectiva de conocimientos en experiencias político-pedagógicas desarrolladas por organizaciones y movimientos sociales, en torno a áreas problemáticas de la vida cotidiana; una de las cuales refiere a la alfabetización de las personas jóvenes y adultas. Luego de ubicar esta problemática, se desarrollan y fundamentan las principales decisiones epistemológicas, teórico-metodológicas y técnicas de esta línea de investigación, así como los primeros avances realizados. Para finalizar, se efectúan reflexiones acerca de la búsqueda de consistencia en relación con los presupuestos generales de la investigación acción participativa y la educación popular, considerados relevantes frente a las acuciantes problemáticas del contexto actual del país y de la región.

Palabras clave: Memoria colectiva. Biografía educativa. Investigación participativa. Alfabetizadores de jóvenes y adultos. Movimientos sociales.

\section{ABSTRACT COLLECTIVE MEMORY AND EDUCATIONAL BIOGRAPHIES OF ALPHABETIZERS IN SOCIAL MOVEMENTS}

This article aims to share methodological aspects of an ongoing line of inquiry, focusing on the challenges of a collective and participatory approach to the reconstruction of the collective memory and 
educational biographies of young and adult's alphabetizers working in organizations and social movements from Argentina. First, the broader research in which this work is framed is presented. This research focuses, through a combined methodological strategy, both qualitative and participatory, on the study of pedagogical and psychosocial processes that account for the collective construction of knowledge, in political-pedagogical experiences developed by organizations and social movements, around problematic areas of everyday life; one of which refers to the literacy of young people and adults. After presenting this problem, the main epistemological, theoretical-methodological and technical decisions of this line of research are developed and grounded, as well as the description of the first results obtained. Finally, reflections are made about the search for consistency of these decisions in relation to the general assumptions of participatory action research and popular education that are considered relevant to the urgent problems of the current context of the country and the region.

Keywords: Collective memory. Educational biographies. Participatory research. Youth and adult literacy teachers. Social movement.

\section{MEMÓRIA COLETIVA E BIOGRAFIAS EDUCATIVAS DE}

\section{ALFABETIZADORES EM MOVIMENTOS SOCIAIS}

Este artigo tem como objetivo compartilhar aspectos metodológicos de uma linha de pesquisa em andamento, enfocando os desafios de uma abordagem coletiva e participativa para reconstruir a memória coletiva e as biografias educacionais de educadores alfabetizadores de jovens e adultos em organizações e movimentos sociais da Argentina. Primeiro, é apresentada a linha de pesquisa geral em que este trabalho está inserido. Esta pesquisa enfoca, por meio de uma estratégia metodológica combinada, qualitativa e participativa, ao estudo dos processos pedagógicos e psicossociais que fazem a construção coletiva do conhecimento, em experiências político-pedagógicas desenvolvidas por organizações e movimentos sociais, em torno de áreas problemáticas da vida cotidiana; uma delas se refere à alfabetização de jovens e adultos. Após a localização desse problema, são desenvolvidas e fundamentadas as principais decisões epistemológicas, teórico-metodológicas e técnicas dessa linha de pesquisa, bem como os primeiros avanços realizados. Por fim, são feitas reflexões sobre a busca da consistência dessas decisões em relação aos pressupostos gerais da pesquisa-ação participativa e da educação popular que são 
considerados relevantes para os problemas urgentes do contexto atual do país e da região.

Palavras clave: Memória coletiva. Biografias Educacionais. Alfabetizadores de jovens e adultos. Movimentos sociais.

\section{Introducción}

Este artículo tiene como objetivo compartir aspectos metodológicos y de avance de una línea de indagación, recientemente iniciada, en torno a la identificación de procesos de construcción de conocimientos en torno a la alfabetización de personas jóvenes y adultas en organizaciones y movimientos sociales, a través de la reconstrucción de la memoria colectiva y de las biografias educativas de alfabetizadores y alfabetizadoras ${ }^{1}$ que realizan su tarea en dichas organizaciones, en Buenos Aires, Argentina.

Se presentará, en primer lugar, la investigación marco en la cual se inscribe esta línea de indagación. En segundo lugar, se expondrán los rasgos principales del área problemática de la alfabetización de las personas jóvenes y adultas, así como de los trabajos antecedentes realizados en relación con la experiencia seleccionada como caso de estudio. Luego se desarrollarán y fundamentarán aspectos metodológicos y técnicos de esta línea de indagación, presentando las primeras tareas de avance realizadas. Se trata de presentar las principales decisiones teórico-metodológicas de esta etapa de la investigación, en tanto dichas decisiones envuelven reflexiones epistemológicas y metodológicas que buscan sostener su consistencia en relación con los presupuestos generales de la Investigación Acción Participativa (IAP) y la Educación Popular.

La línea de trabajo en la cual se profundiza

1 Se acuerda con la utilización de lenguaje inclusivo. Sin embargo, dada la publicación de este texto, en español, en una revista brasileña, se opta por continuar con las denominaciones tradicionales, sólo a efectos de facilitar la lectura. en este artículo forma parte de un proyecto de investigación marco ${ }^{2}$, orientado a indagar en los procesos pedagógicos y psicosociales que dan cuenta de la construcción colectiva de conocimientos, en experiencias político-pedagógicas de diferente grado de formalización ${ }^{3}$, en organizaciones y movimientos sociales, ámbitos sociocomunitarios. Se trata de un estudio de casos en relación con áreas problemáticas de la vida cotidiana (tales como, vivienda y hábitat; participación social y política; alfabetización y educación de jóvenes y adultos; salud).

El interés por este objeto de estudio tiene su anclaje en el devenir sociohistórico y actual

2 En este artículo se hará referencia a esta investigación mayor como "investigación marco", en tanto involucra a ésta y a otras líneas de trabajo. La realización de esta investigación marco, dirigida por S. Llosa, ha sido aprobada y sostenida a través de un subsidio de la Universidad de Buenos Aires (UBACYT 2018-2020). Tiene su sede en el Instituto de Investigaciones en Ciencias de la Educación IICE-UBA. Esta investigación marco incluye también la realización de dos tesis de maestría, una de doctorado y otra de postdoctorado.

3 Desde una perspectiva amplia de la Educación a lo largo de la vida, se incluye la multiplicidad de experiencias educativas ya sea que acontezcan en la escuela o más allá de la escuela. Se discrimina entre tres componentes: la Educación Inicial incluye fundamentalmente al sistema educativo formal en todos sus niveles (desde el nivel preescolar al universitario); la Educación de Jóvenes y Adultos, refiere al conjunto de actividades educacionales dirigidas a la población de 15 años y más, ya sea organizadas para completar los niveles educativos o a la formación en las diferentes áreas (trabajo, salud, participación, etc.); y los Aprendizajes Sociales, refieren a aquellos difusos e inestructurados ámbitos de aprendizaje por los que atravesamos en nuestra vida cotidiana. Estos tres componentes pueden ser analizados según su formalización, en cuanto a la organización y estructuración de las actividades educativas en sus distintas dimensiones y aspectos; esta noción de grados y tipos de formalización intenta superar las limitaciones de los tradicionales conceptos de educación formal / educación no formal / educación informal (SIRVENT, TOUBES, SANTOS, LLOSA Y LOMAGNO, 2010). 
de Argentina. En los últimos años, desde nuestras experiencias de docencia, investigación y extensión universitaria, nos sorprende, interpela e interroga la emergencia de un fuerte reconocimiento y aún, reivindicación por parte de algunas organizaciones sociales, respecto de los "saberes acumulados", de los conocimientos construidos a partir de los aprendizajes sociales y del entrelazamiento de múltiples saberes, en diversos espacios de la vida organizacional. Esta disposición contrasta con la situación anterior, cuando, durante la década de 1990, con la consolidación del modelo neoconservador y neoliberal bajo el patrón de dominación de la "nueva derecha" (MENGUINI, MIGLIAVACCA Y MAS ROCHA, 2016), se agudizaron las múltiples pobrezas y los factores inhibitorios de la participación y de la organización social (SIRVENT, 2008). Se observaba, en esa época, entre otras cuestiones, la dificultad para reconocer los aprendizajes vividos y los saberes construidos en las organizaciones sociocomunitarias barriales.

En Argentina, esta crisis tuvo su máxima expresión a fines de 2001, momento de profundos conflictos socioeconómicos y políticos y de cambios, a partir del cual se reavivaron y/o fortalecieron ciertas experiencias organizativas y aún se generan otras nuevas formas de organización y movilización popular articuladas en el devenir de procesos de construcción de demandas sociales en pos de la realización de diversas necesidades y derechos. Al mismo tiempo, sin embargo, se continuaron ciertos fenómenos y formas de ejercicio de poder inhibitorios (tales como la fragmentación o el clientelismo político), en la permanente contradicción entre estos aspectos inhibitorios y otros que, en cambio, aportaban al crecimiento en la conciencia y defensa de los derechos humanos y sociales (SIRVENT, 2008; SIRVENT Y LLOSA, 2006). Las organizaciones sociales han ido asumiendo complejas formas de relación con el Estado, desde el rechazo o la disputa, hasta formas de asimilación (MALDONADO, 2016).

De manera entramada con estos procesos, diversas organizaciones y movimientos sociales han ido reconociendo el componente pedagógico involucrado en sus prácticas cotidianas y han avanzado en la generación y/o recreación de estrategias educativas, con una orientación político-pedagógica que podríamos ubicar de manera general, en una perspectiva crítica, desde una mirada amplia de lo educativo y con intencionalidad transformadora ${ }^{4}$. En estos nuevos actores sociales "[...] se percibe [...] un reconocimiento explícito del relevante lugar de la educación en su proceso de organización [...] y de la capacidad de construcción colectiva de conocimiento [...]" (RIGAL, 2008, p. 34). Distintas instituciones barriales y organizaciones sociales buscan sistematizar las experiencias y los conocimientos logrados; y aún más: buscan instituir espacios de formación de formadores para transmitir dichos conocimientos. La existencia de estos procesos, que aún constituyen un campo de estudio "incipiente" (BARALDO, 2010) o "en construcción" (GUELMAN, 2011), alienta a indagar en la dimensión pedagógica, en la "caja negra" de los procesos que puedan dar cuenta de dicha producción de conocimiento.

En esa dirección se encamina la investigación marco. Los objetivos se orientan a generar categorías analíticas que permitan identificar, analizar y comprender aquellas experiencias valiosas y potentes en términos de construcción colectiva de conocimientos, en un sentido transformador. Esto implica, también para el

4 Varias investigaciones han permitido construir un cierto consenso respecto del potencial formativo de la experiencia cotidiana de las organizaciones y movimientos sociales en varios países de la región, en cuyo marco se inscriben también acciones intencionalmente educativas (GHON, 1999; ZIBECHI, 2008; MICHI, DI MATTEO Y VILA, 2012; GUELMAN, 2011; RIGAL, 2008; VILLAGRA, ZINGER Y PATAGUA, 2015; entre otros). 
equipo investigador, una intencionalidad teórica y política, comprometida con horizontes de emancipación y transformación social.

La estrategia metodológica elegida se concreta en un diseño combinado, de tipo cualitativo y participativo, que supone la combinación de diferentes modos de hacer ciencia de lo social (SIRVENT Y RIGAL, 2016). El modo de generación de conceptos, desde el cual se enfatiza la perspectiva cualitativa e interpretativa, se interesa por los significados que las personas les atribuyen a los sucesos, a sus acciones e interacciones, dentro de un contexto histórico y social determinado. Y el modo participativo, desde el cual se enfatizan aspectos epistemológicos del paradigma de la teoría social crítica, se orienta a generar procesos de participación real de la población objeto de estudio, en la construcción colectiva de un conocimiento científico, como instrumento para una praxis social emancipatoria. Se busca el crecimiento de la población en su capacidad de participación y construcción de poder, a través de la articulación entre investigación, participación y educación; en este sentido, supone un entramado dialéctico entre las prácticas de la IAP y las acciones de Educación Popular (SIRVENT, 2011; SIRVENT, LLOSA Y STEIN, 2018).

El diseño de esta investigación marco se concreta a través de un estudio de casos, en cuanto a la selección de aquellas experiencias político-pedagógicas desarrolladas en el marco de organizaciones y movimientos sociales, en las cuales se desarrollen procesos de construcción colectiva de conocimiento, en torno a áreas que estas organizaciones consideren problemáticas a enfrentar y superar, tales como la alfabetización y educación de jóvenes y adultos; la participación social y formación política; el territorio, la vivienda y el hábitat.

En la primera de dichas problemáticas se inscribe la línea de indagación sobre la cual se profundiza en este artículo.
La alfabetización y la formación de alfabetizadores como problemática para la investigación, la acción y la participación

Se asume una perspectiva conceptual amplia, global e integral del fenómeno educativo, que conlleva incluir a la alfabetización de personas jóvenes y adultas en un continuo de educación a lo largo de la vida. Desde esta perspectiva, se enfatiza el reconocimiento de la educación como una necesidad y un derecho humano y social para todas las personas, a lo largo de toda la vida. Sin embargo, la realización de esta necesidad y el cumplimiento de este derecho no están garantizados para todos por igual. Para muchos jóvenes y adultos de nuestro país, la educación es una carencia más en un mundo de pobrezas que tiende a reproducirse, asociada a la desigualdad entre clases sociales, así como a situaciones de discriminación e injusticia social.

Según el censo nacional INDEC de 2010, en Argentina se registran 528.349 personas mayores de 15 años que nunca asistieron a la escuela, lo cual representa el $1,8 \%$ de la población. Estos datos se agravan, según investigaciones antecedentes, al considerar que, de las personas de 15 años y más que alguna vez asistieron pero que ya no asisten a la escuela, el $12,8 \%$ posee como máximo nivel educativo alcanzado sólo el primario incompleto; se trata de 3.117.102 personas. Los análisis muestran su asociación principal con la situación de pobreza (medida a través de las Necesidades Básicas Insatisfechas NBI) y con las diferencias regionales dentro del país (SIRVENT Y LLOSA, 2012). Se trata de los escalones de mayor vulnerabilidad y de vulneración de derechos, en el marco de una situación educativa de riesgo ${ }^{5}$.

5 El concepto de Nivel Educativo de Riesgo (SIRVENT, 1997) refiere a la probabilidad estadística que tiene 
Aunque la Argentina sea considerada dentro de los parámetros internacionales con altos índices de alfabetización, esta perspectiva varía según a qué se considere estar alfabetizado. El concepto de alfabetismo ha sido gradualmente ampliado. Desde una perspectiva cualitativa, la noción de alfabetismo hace referencia a la compleja red de conocimientos que un ciudadano necesita para analizar crítica y autónomamente los hechos de su entorno barrial, regional e internacional; ser alfabeto en la actualidad, supera ampliamente la adquisición de los instrumentos rudimentarios para la lectura y la escritura (SIRVENT Y LLOSA, 2001).

Este diagnóstico cuantitativo y cualitativo de vulneración de derechos se encarna en la vida cotidiana en los territorios populares urbanos, al afectar especialmente a las personas jóvenes y adultas de los sectores populares, impactando incluso sobre las posibilidades de la participación social:

Nos dimos cuenta que había compañeros del movimiento que no podían leer nuestros materiales que se necesitaban para tomar algunas de las tareas militantes. (Alfabetizadora de uno de los movimientos sociales en reunión del día $12 / 05 / 2016)$

En este contexto, varias organizaciones y movimientos sociales no sólo han expresado sus demandas al Estado en tanto responsable y garante del derecho a la educación, sino que han adoptado y construido propuestas de alfabetización para la población más vulnerada, que por distintas razones no ha podido acceder a otras opciones educativas. Para ello, han organizado campañas y/o centros de alfabetización en distintas sedes de los propios movimientos y/o en otras organizaciones barriales

un conjunto de población de quedar marginado de la vida social, política y económica según el nivel de educación formal alcanzado. Su dimensión cuantitativa refiere, en términos operacionales, a la población de 15 años y más que no asiste a la escuela, cuyo nivel educativo alcanzado es menor o igual al nivel secundario incompleto. (comedores comunitarios, centros de jubilados, etc.) o, aún, en espacios cedidos en viviendas de los propios vecinos ${ }^{6}$. Y han realizado, asimismo, convocatorias para quienes quieran desempeñarse como alfabetizadores. Los alfabetizadores, en consecuencia, son tanto educadores militantes de los propios movimientos como otros "voluntarios", que se suman a la tarea. Si bien algunos tienen formación docente específica, muchos otros son estudiantes de nivel secundario, de profesorados o de distintas carreras universitarias. Los propios referentes alfabetizadores de los movimientos suelen proveer una breve formación inicial a quienes se acercan, pero esto no agota la búsqueda de nuevas oportunidades y fuentes de formación.

En esas búsquedas, en 2015, varios alfabetizadores participaron de un curso de formación docente, basado en los resultados de maestría y doctorado de una de las integrantes del equipo de esta investigación sustentados en una concepción de Educación Permanente y de Educación Popular y en una Epistemología Psicogenética Constructivista (KURLAT, 2011, 2014). A partir de estos primeros intercambios, se ha ido descubriendo cómo las organizaciones y movimientos populares han tomado esta problemática como una de sus áreas de acción colectiva y aún más, cómo han encontrado en la alfabetización y educación popular una herramienta para la intervención militante. Al mismo tiempo, se fueron identificando nudos críticos referidos a sus necesidades de formación, así como, de generación de intervenciones pedagógico-didácticas fundadas en una metodología acorde, adaptadas a las características del aprendizaje de las personas

6 Ejemplos de ello son: las propuestas de creación de Escuelas Primarias Populares y la implementación de Campañas de Alfabetización desde el Movimiento Popular La Dignidad "El futuro es nuestro"; la Campaña "Del papel al poder", desde la Corriente de Organizaciones de Base (COB La Brecha); la conformación de espacios de alfabetización del Frente Arde Rojo, entre otras. 
jóvenes y adultas y a la especificidad de sus espacios de alfabetización.

Esa fue la semilla de un camino compartido, que aún estamos recorriendo, a través de una trama que articula, hasta el presente, diversos dispositivos de investigación, docencia y extensión universitaria, implicando un trabajo conjunto entre docentes-investigadores universitarios y referentes de alfabetización y alfabetizadores que desarrollan propuestas de alfabetización de personas jóvenes y adultas, así como, de formación de alfabetizadores en sus organizaciones y movimientos sociales?.

En el año 2016, este trabajo conjunto comenzó a tejerse en relación con espacios académicos de formación de grado en Ciencias de la Educación de la Facultad de Filosofia y Letras de la Universidad de Buenos Aires. Posteriormente, desde 2017 y hasta la actualidad, la experiencia tomó forma a través de Proyectos de Extensión Universitaria ${ }^{8}$, orientados al fortalecimiento de los procesos de alfabetización de personas jóvenes y adultas en organizaciones y movimientos sociales. Los principales dispositivos de trabajo implementados son?:

- La realización de observaciones, registros y análisis de las prácticas alfabetizadoras; esta tarea, inicialmente desarrollada por estudiantes universitarios, fue paulatinamente realizada

7 A lo largo del camino han participado en los sucesivos Proyectos de Extensión, alfabetizadores y alfabetizadoras integrantes de diferentes anclajes territoriales de las siguientes organizaciones y movimientos sociales: Movimiento Popular La Dignidad, COB La Brecha, Frente de Organizaciones en Lucha, Frente Arde Rojo, Casa de la Cultura Compadres del Horizonte, La Poderosa, Movimiento Campesino de Córdoba. Las prácticas alfabetizadoras suelen organizarse por zonas, con la coordinación de un "referente alfabetizador".

8 Dichos Proyectos de Extensión Universitaria (denominados UBANEX a nivel institucional), dirigidos por Llosa y Kurlat, han sido seleccionados para ser sostenidos por la Universidad de Buenos Aires, a través de sucesivos subsidios, durante los años 2017, 2018 y 2019.

9 Para más detalles ver en KURLAT Y LLOSA, 2019a; 2019b. por los propios referentes alfabetizadores. Los resultados de los análisis fueron compartidos en sucesivas reuniones de retroalimentación, con la participación de integrantes de las organizaciones y movimientos sociales involucrados.

- La organización de talleres de construcción colectiva de secuencias y de proyectos didácticos para la alfabetización de personas jóvenes y adultas.

- La implementación de otras propuestas de fortalecimiento de las prácticas alfabetizadoras: "mesa de libros itinerante", "cineclub", publicación de materiales didácticos ("cuadernillos"), producto del trabajo colectivo realizado.

Como parte de este camino compartido, también se ha implementado, en 2018, un seminario-taller de extensión universitaria, a efectos de profundizar la formación. En la planificación de dicho seminario participaron los referentes de alfabetización, quienes resaltaron, entre otras cuestiones, su interés en torno a las biografías educativas de los alfabetizandos: "Es importante ver lo de las biografias educativas; con eso entendés quiénes son y lo que les pasa a las personas que se acercan a nuestros espacios"10.

Esto reforzó la inclusión de contenidos al respecto, sobre la base de investigaciones anteriores (LLOSA, 2012, 2017).

A lo largo de todo este trabajo se fueron conformando una serie de aspectos que, finalmente, hoy pueden interpretarse como condiciones potentes para iniciar un nuevo camino, de participación de estos alfabetizadores, en la investigación sobre los procesos de construcción colectiva de conocimiento. Dichos aspectos refieren a:

10 Narrativa generada en la reunión de planificación colectiva del Seminario-taller de Extensión Universitaria, realizada el 18/04/2018. 
- la identificación de evidencias sobre la existencia de procesos previos de producción de conocimiento en torno a sus prácticas alfabetizadoras;

- la paulatina conformación de un grupo de trabajo, con los alfabetizadores, en cuanto a su involucramiento con las tareas desarrolladas a lo largo del tiempo y en cuanto a la emergencia de vínculos de reconocimiento, confianza y afecto, no sólo con el equipo investigador de la universidad, sino entre alfabetizadores integrantes de las distintas organizaciones sociales involucradas; esto resulta significativo dado que, en la IAP, lo grupal, tiene una centralidad metodológica en tanto instancia productora de un conocimiento colectivo y científico (SIRVENT Y RIGAL, 2016);

- la apropiación, por parte de los alfabetizadores, de ciertos posicionamientos conceptuales y metodológicos compartidos con el equipo investigador de la universidad, a lo largo del desarrollo de la experiencia, en torno a la alfabetización;

- la apropiación y valoración de técnicas propias del quehacer investigativo, por parte de los alfabetizadores; incluso realizando observaciones "cruzadas" entre las distintas organizaciones participantes;

- la apropiación y valoración del abordaje biográfico, a partir del intercambio sobre conceptos y aspectos metodológicos básicos de nuestras investigaciones anteriores y sobre sus propias vivencias devenidas de comenzar a implementar proyectos didácticos con la incorporación de narrativas biográficas de los alfabetizandos como estímulo para la lectoescritura.

\section{Organización social, memoria colectiva y biografía educativa}

A la luz de la tríada entre los objetivos generales, el objeto y el problema de investigación planteados en el proyecto de investigación marco (presentados en el primer apartado de este artículo), el camino previo compartido con los alfabetizadores en el proyecto de extensión universitaria y las condiciones facilitadoras identificadas condujeron a focalizar, como uno de los estudios de caso ${ }^{11}$, en los procesos de construcción colectiva de conocimientos en torno a la alfabetización de personas jóvenes y adultas, en las experiencias de las organizaciones y movimientos sociales con las que veníamos trabajando ${ }^{12}$.

De esta focalización emergen varias líneas de trabajo. A efectos de este artículo, se presenta la línea de indagación en relación con los siguientes interrogantes generales ¿Cómo ha sido el devenir de los procesos de construcción colectiva de conocimientos relativos a las prácticas alfabetizadoras en organizaciones y movimientos sociales? ¿Qué aspectos y procesos pedagógicos y psicosociales pueden dar cuenta de ese devenir?

Estas preguntas han alentado a un abordaje histórico - biográfico, apropiado tanto a la naturaleza del objeto de estudio, como a los posicionamientos epistemológicos, metodológicos y éticos relativos a la IAP asumidos. Se ha comenzado entonces (tal como se detallará más abajo), compartiendo y acordando una propuesta de trabajo, con los referentes de al-

11 Actualmente el proyecto de investigación marco UBACYT se despliega en relación con éste y otros casos seleccionados, a efectos comparativos buscando generar categorías teóricas de mayor alcance. Los otros casos (en distintos estadíos de avance) refieren a las problemáticas de la vivienda y el hábitat, a las problemáticas de la participación social y política y a las problemáticas ambientales.

12 Estamos trabajando con tres organizaciones sociales; dado que se trata de un momento inicial de trabajo compartido, se prefiere conservar el anonimato, previendo en un futuro arribar a autorías colectivas. 
fabetización de las organizaciones y movimientos sociales participantes en el proyecto de extensión. Se han ido delineando interrogantes específicos: ¿Cuándo y cómo se ha construido conocimiento acerca de la problemática y de las necesidades de alfabetización y educación de personas jóvenes y adultas? ¿Cuáles han sido los momentos clave y los estímulos que han operado en la construcción colectiva de conocimientos, a lo largo del tiempo? ¿Cuáles han sido las fuentes de construcción de conocimiento? ¿Cuáles han sido las trayectorias de formación de los alfabetizadores, en tanto protagonistas portadores y constructores de conocimientos?

Se ha tornado relevante la búsqueda de propuestas metodológicas que sean consistentes con dichos interrogantes y adecuados a las características del caso seleccionado.

En tal sentido, se destacan algunos autores que han observado la contribución del uso de historias de vida individuales y de materiales biográficos a las investigaciones sobre movimientos sociales (entre otros, SALTALAMACCHIA, COLÓN, RODRÍGUEZ, 1983; TORRES CARRILLO, 2010). Saltalamacchia, Colón y Rodríguez (1983) han entendido por "movimiento social" a una configuración social limitada, constituida en torno a una lucha por una determinada reestructuración de poder, que supone la aparición de interrelaciones entre un conjunto de individuos y la constitución de una identidad colectiva sostenida en una cosmovisión compartida. Señalan que su carácter "contestatario" y, aún, la represión gubernamental, atentan contra la existencia y/o la sobrevivencia de rastros documentales, no registrados por los archivos oficiales. Teniendo en cuenta, además, que la conformación y organización de estos movimientos se desarrolla en interjuego con una historia nacional y regional conflictiva, en complejos procesos de disgregación, fusión y reestructuración de relaciones entre sujetos e instituciones, estos autores resaltan los beneficios de recurrir a materiales biográficos, ya sea para mapear las instituciones y organizaciones en las que se fue forjando el movimiento social, para identificar informaciones factuales que hacen a su desarrollo temporal $y$, especialmente, para captar las vivencias significativas y para reconstruir el sentido de las acciones colectivas.

La solidaridad que lleva a la acción colectiva a ciertos individuos [...] nace, en el plano subjetivo, a partir de la experiencia de encuentro y reconocimiento provocados por la vivencia de ciertos acontecimientos. Cuáles son esas vivencias y cómo fueron interpretadas en su momento es algo que comúnmente se pierde en las fuentes escritas que es posible encontrar a posteriori. La historia de vida puede ser una forma adecuada para reconstruirlas. (SALTALAMACCHIA, COLÓN Y RODRÍGUEZ, 1983, p. 325).

Los abordajes metodológico-biográficos se han utilizado en diversas disciplinas de las ciencias sociales y humanas desde larga data, haciendo énfasis principalmente en una dimensión individual. Aún cuando es posible desarrollar relatos de vida apelando, de manera instrumental, a intercambios grupales, estos quedan fundamentalmente centrados en la experiencia individual. Sin embargo, tal como repara Le Grand, "[...] la dimensión colectiva está ahí presente" (2004, p. 7): en los relatos, aparece un conjunto entrelazado de estructuras familiares, institucionales y profesionales de pertenencia, de itinerarios, relacionados con trayectorias comunes a una generación, de experiencias compartidas. "El otro y los otros están siempre presentes, por todas partes" (LE GRAND, 2004, p. 8).

La existencia de una dimensión colectiva en toda producción biográfica (aún aquella que pueda pensarse como más personal, íntima y "única"), resulta una invitación a una mirada y a una producción colectivas. En ese 
caso, se supera la sumatoria de los testimonios individuales como fuente para reconstruir lo colectivo, adquiriendo una especificidad y una denominación propia. Le Grand propone reservar el término "historia de vida colectiva" para aquellas situaciones en las que existe "[...] una entidad colectiva con una experiencia común, en el seno de una vida colectiva real. Dicho de otra manera, allá en donde existe una colectividad de pertenencia y no solamente una comunidad de destino [...]" (2004, p. 6). Rhéaume, por su parte, haciendo hincapié en una aproximación biográfico narrativa para la investigación-acción-intervención comunitaria, también destaca la particularidad, la complejidad y la implicación relativa al "relato de vida colectivo", definido como "[...] el relato de un colectivo que elabora una palabra en conjunto, en relación con su historia vivida en tanto que grupo o colectivo instituido [...]" (2002, p. 113); refiere a la historia narrada producida por el grupo en encuentros, acerca del surgimiento y la constitución del grupo, su evolución, sus realizaciones, sus proyectos futuros.

La reconstrucción de la memoria colectiva, por otro lado, ha sido una estrategia de producción de conocimiento de la educación popular (TORRES CARRILLO, 2010). En tanto proceso y producto de prácticas biográficas colectivas, permite resaltar la relación entre la experiencia personal y la historia producida; responde a una necesidad vital de los individuos y de los grupos, ligada al trabajo sobre la identidad colectiva, a la elaboración de duelos y conflictos, a la constitución de una base para la elaboración de proyectos compartidos. Tal como señala Le Grand "[...] sin duda alguna, contar la historia es una forma de poder [...]" (2004, p. 7).

Los autores remarcan los efectos formativos de este tipo de prácticas biográficas colectivas, así como la relevancia de que dichas prácticas sean participativas (RHÉAUME, 2002;
LE GRAND, 2004); una metodología reflexiva de Educación Popular puede proyectarlas hacia una elaboración cultural y educativa (LE GRAND, 2004). El anclaje en grupos que hayan constituido o constituyan un colectivo, las tornan una herramienta potente de trabajo con organizaciones y movimientos sociales.

Teniendo en cuenta estas cuestiones, en la línea de investigación presentada en este artículo se asume entonces al enfoque o método biográfico de manera amplia, en tanto “[...] el conjunto de los procedimientos seguidos para organizar la investigación alrededor de un yo individual o colectivo que toma forma narrativa incorporando sus descripciones de experiencias y sucesos y sus interpretaciones [...]" (SAUTU, 1999; p. 23; destacado propio). El caso seleccionado para su estudio, ha conducido a la búsqueda y elaboración de estrategias metodológicas consistentes, que enfatizaran lo grupal.

Por un lado, desde el plan de trabajo de la investigación marco, el objeto problema planteado en torno a procesos colectivos, condujo a prever el desafio de la combinación de unidades de análisis colectivas e individuales. Buscando la coherencia con el enmarcamiento conceptual y metodológico sostenido, entre otras cuestiones, en supuestos centrales de la Educación Popular y de la IAP, se subraya la relevancia de lo grupal y de los procesos de reconstrucción de la memoria colectiva, en su impacto en la construcción de identidad y de poder; es decir, se asume el valor político que implica este tipo de procesos investigativos.

Por otro lado, tal como se ha mencionado más arriba, el trabajo previo realizado ha permitido avizorar no sólo la conformación de un grupo de base constituido por los investigadores universitarios y los referentes alfabetizadores, sino su interés por continuar trabajando juntos y por incorporar el trabajo biográfico. En las reuniones en las que acordamos el 
tipo de trabajo investigativo a desarrollar, el énfasis en lo colectivo quedó expresado por los participantes:

Me parece muy interesante eso de ir recuperando una memoria colectiva, pero desde los colectivos en sí, no de ir desde lo individual, que bueno, agarrás un par de personas y hacés entrevistas por cada lado, de cada individualidad y después vas tratando de unir lo común. Querría el proceso inverso, de ir desde lo colectivo $y$ lo general, a lo individual ${ }^{13}$.

Por otra parte, en los trabajos antecedentes se sugiere, además, la pertinencia y utilidad de una combinación de técnicas y fuentes de datos. González Terrero, Aguilera Morales y Torres Carrillo (2013), por ejemplo, han abordado la investigación de la producción de subjetividades y la formación de sujetos en y con organizaciones y movimientos sociales, a través de una perspectiva metodológica participativa y crítica, incluyendo como uno de sus momentos investigativos a la reconstrucción descriptiva y narrativa de los procesos y trayectorias organizativas; para ello han incluido revisiones documentales, activación colectiva de la memoria, encuentros pedagógicos y afectivos en reuniones con integrantes pasados y presentes, así como biografias individuales (a través de historias y relatos de vida y de entrevistas), entre otras técnicas y estrategias.

En el caso de la línea de investigación que aquí se presenta, los interrogantes han conducido a la combinación de técnicas grupales para la reconstrucción de la memoria colectiva con técnicas orientadas a la indagación en las biografias educativas personales de los alfabetizadores participantes. En efecto, el estudio de la construcción colectiva de conocimiento supone el desafio de abordar un objeto complejo, en el cual se interrelacionan fuentes y portadores de diferentes saberes, en las tramas entre lo individual y lo colectivo.

13 Expresión de uno de los alfabetizadores, en la reunión realizada el 09/08/2019.
Teniendo en cuenta el énfasis de nuestra investigación en unidades de análisis colectivas, el trabajo sobre las biografías educativas personales ha sido planteado como complementario; tal como señala Rhéaume (2002), los relatos de vida individuales pueden complementar el relato colectivo, permitiendo esclarecer más la historia del grupo, así como comprender los significados que los integrantes le asignan a sus momentos clave.

En este caso, al trabajar con alfabetizadores de personas jóvenes y adultas que actúan en organizaciones y movimientos sociales, la complejidad se profundiza; se trata de un campo poco explorado en su especificidad, en cuanto a sus muy diversas trayectorias de formación en torno a la alfabetización, las cuales frecuentemente se han conformado a través del tránsito por espacios educativos de un bajo grado de formalización (talleres, jornadas de encuentro, intercambios informales, trabajo autodidacta, etc.). Es relevante entonces poder reconstruir las fuentes y las trayectorias desde las cuales se aporta a la construcción de conocimiento colectivo.

El enfoque de las biografías educativas supone abordar la totalidad de las fuentes de formación y la interacción entre aprendizajes construidos en diferentes espacios educativos y en experiencias cotidianas, como una globalidad que se despliega a lo largo de toda la vida. Esta perspectiva, desarrollada en varias investigaciones antecedentes (entre otros, DOMINICÉ, 1988, 1990; DE VILLERS, 1990; ALHEIT, 1995; JOSSO, 1999; LLOSA, 2016, 2017), también se orienta hacia la búsqueda de la emancipación personal y social del sujeto y hacia la democratización del conocimiento, trascendiendo su utilización como instrumento de relevamiento de información empírica en la investigación hacia la búsqueda de un impacto en la formación de los propios narradores. Varios trabajos han mostrado, además, la riqueza de 
la inclusión de espacios grupales, en los cuales los entrevistados pueden reflexionar sobre sus biografias educativas, en interjuego con las interpretaciones de otros narradores así como las del propio investigador (DOMINICÉ, 1988; SOUZA, 2007; PASSEGGI, 2009, entre otros) ${ }^{14}$.

En la línea de investigación aquí presentada, teniendo en cuenta las consideraciones efectuadas más arriba, se opta también por un tratamiento grupal de las biografias educativas. En este sentido, resulta inspiradora la propuesta de los "Círculos Reflexivo Biográficos" desarrollada por E. Olinda y su equipo, por su carácter innovador, al proponer un dispositivo de trabajo basado, entre otras fuentes, en la lógica de los "círculos de cultura" freireanos, que se desarrolla en reuniones grupales sucesivas, en las cuales se trabajan los acuerdos iniciales con el grupo, su sensibilización hacia el trabajo biográfico y se implementan diversos recursos reflexivos, expresivos y creativos (ver por ejemplo, en OLINDA Y PINTO, 2019).

En síntesis, buscando la consistencia con los supuestos epistemológicos, teóricos y metodológicos que se asumen en esta línea de investigación, se ha abrevado entonces en autores que, de manera general, han enfatizado la potencia del trabajo grupal para el abordaje del devenir tanto de una organización colectiva como de una trayectoria educativa personal reconociendo y asumiendo los aportes de la educación popular y del involucramiento participativo de los sujetos en la investigación, propiciando procesos reflexivos y críticos en una dirección emancipatoria.

\section{La propuesta metodológica elaborada: avances y perspectivas de continuidad}

14 Desde otra perspectiva de trabajo se desarrollan experiencias pedagógicas realizadas desde un enfoque biográfico narrativo, en redes y colectivos docentes (por ej., ver en SUÁREZ, 2015).
La línea de investigación acerca del devenir de los procesos de construcción colectiva de conocimientos en torno a las prácticas alfabetizadoras en organizaciones y movimientos sociales ha comenzado con un momento inicial de trabajo, dedicado al logro de los primeros acuerdos en torno a la participación del grupo de referentes y de alfabetizadores en la investigación y al reajuste del plan de trabajo, en reuniones donde se amplió la información básica que este grupo ya disponía acerca del proyecto de investigación marco y se formularon preguntas específicas para esta línea de indagación. Luego se planteó, de manera general, la propuesta metodológica, referida a la reconstrucción colectiva histórico-biográfica, y sus fundamentos $^{15}$. Los participantes fueron manifestando, a su vez, sus interrogantes y sus opiniones; y manifestaron su interés por formar parte activa del proceso de investigación, esbozando desde un primer momento una identificación de posibles fuentes y modos de acceso a la información. Estas son algunas de sus expresiones:

“Esto es muy interesante!"

"Para mí está buenísimo; siento que es un montón de laburo..."

"Igual para mí es muy posible, eh? porque, aunque dudo que algunos compañeros puedan venir a una reunión acá, podemos hacer entrevistas cuando nos encontramos en los encuentros del movimiento o en los espacios de alfabetización"16.

Se ha sistematizado las expectativas de los integrantes del grupo y ellos han resignificado y se han apropiado de esta línea de la investigación, repensando el propósito de profundizar los espacios participativos en la

15 Los acuerdos para la realización de este trabajo trascienden a los integrantes del propio grupo, dado que ellos deben, a su vez, refrendar estos acuerdos con cada una de sus organizaciones.

16 Fragmento de registro de las expresiones de los referentes alfabetizadores en la reunión donde se trabajó colectivamente la propuesta de trabajo, realizada el día 21/07/2019. 
investigación y la utilidad de la generación de conocimiento para sus propias prácticas, en términos de:

- conocer más las propias organizaciones de pertenencia y resguardar su memoria;

- aprender de otras y otros; crecer individual y colectivamente;

- construir un material para compartir con los militantes en los distintos espacios de la organización, así como para la formación de otros voluntarios que se acerquen a alfabetizar;

- instalar la problemática de la alfabetización a nivel más amplio, para avanzar en su resolución y potenciar las prácticas alfabetizadoras.

Sobre esta base inicial de acuerdos y propósitos compartidos, se previó un plan de trabajo que se despliega en tres estrategias metodológicas interrelacionadas, las cuales implican, a su vez, una combinación de técnicas:

a) Reconstrucción de la memoria colectiva; esto abarcaría:

- La reconstrucción de la memoria colectiva acerca de las prácticas alfabetizadoras desarrolladas por cada una de las organizaciones y movimientos sociales a lo largo del tiempo, a través de la elaboración de historias colectivas y de entrevistas.

- La elaboración de la historia colectiva compartida previamente por este grupo, cuyos integrantes son referentes de dichas organizaciones.

b) Elaboración de las biografias educativas de los integrantes del grupo

c) Integración de la memoria colectiva y biográfica producto de las estrategias anteriores, en reuniones de retroalimentación ampliadas.

Actualmente se está desarrollando la primera de las estrategias arriba mencionadas, dirigida a la reconstrucción de la memoria colectiva. Esta estrategia se orienta a la identificación de los hitos principales que han marcado el devenir de las prácticas alfabetizadoras en el tiempo, así como de los momentos clave de la construcción de conocimiento en torno a éstas.

Dicha estrategia se despliega, motoriza y direcciona a través de una serie de reuniones, en las cuales, al mismo tiempo, se va constituyendo también el grupo investigador, con la participación de integrantes alfabetizadores de las organizaciones sociales, profundizando los vínculos previos, en la interrelación y la toma de decisiones conjuntas.

Los participantes alfabetizadores han iniciado una identificación de diferentes fuentes de información que incluyen documentos escritos, publicaciones digitales, fotos, mensajes en redes sociales. Así como han propuesto realizar entrevistas a otros informantes clave; es interesante señalar que, entre estos informantes clave, han incluido no sólo a quienes fueron alfabetizadores referentes históricos de cada organización que no están participando actualmente del grupo, sino que señalaron la relevancia de entrevistar también a los referentes territoriales que viven en los barrios y que habilitan y organizan los espacios comunitarios para que la tarea alfabetizadora se realice.

De esta manera, las decisiones colectivas y la dinámica grupal, ha conducido a iniciar el trabajo con la elaboración de la historia de vida colectiva en torno a las prácticas alfabetizadoras desarrolladas por uno de los movimientos sociales participantes, a través de la aplicación de varias técnicas de relevamiento de información: búsqueda de documentos; relatos de vida colectiva; intercambio sobre materiales evocadores; entrevistas semiestructuradas a otros informantes clave.

Se ha trabajado con ellos la elaboración de ejes temáticos como guía para la realización 
de las entrevistas, así como se han compartido los principales aspectos técnicos. Se ha reflexionado, en particular, acerca de que la realización de las entrevistas por parte de estos alfabetizadores hacia otros alfabetizadores, quienes son, a su vez, sus propios compañeros de tareas, colabora con "reducir al mínimo la violencia simbólica que puede ejercerse" a través de una entrevista" (BOURDIEU, 1993, p. 529), dada la proximidad social y la familiaridad entre ellos; pero que, sin embargo, no por ello no está exento de desafíos, especialmente en términos de quebrar la tendencia a la naturalización y a la mitificación del pasado compartido.

En las reuniones grupales se ha estimulado el relato de vida colectiva, a través de preguntas clave y del trabajo con materiales evocadores provistos por los mismos alfabetizadores (volantes, afiches de convocatorias a alfabetizadores voluntarios, distintos logos utilizados, etc.). Cada pregunta y/o material expuesto desata un rico ida y vuelta de intervenciones de los participantes, con aportes, repreguntas, rectificaciones, ampliaciones, debates entre distintos puntos de vista...

La activación de la memoria colectiva realizada hasta el momento ha permitido la elaboración colectiva de una primera cronología, sobre una línea de tiempo, en la cual se identificó, de manera preliminar:

- el inicio de las prácticas alfabetizadoras, cuando se identificaron las necesidades y demandas de militantes y de habitantes de los barrios, ligadas a la lectoescritura

- los principales hitos o momentos clave relativos al devenir de las prácticas alfabetizadoras y de la formación de alfabetizadores

- la identificación y ubicación temporal de la acción de los principales referentes alfabetizadores

- la influencia de la constitución del pro- pio grupo a través de las acciones del equipo investigador-educador de la UBA, en el proyecto de extensión universitaria.

- primeras relaciones entre el devenir de las prácticas alfabetizadoras y el de la organización social como un todo, así como la influencia de aspectos del contexto sociohistórico.

- algunas pistas o evidencias sobre los principales procesos de construcción colectiva de conocimientos

Cada reunión ha sido planificada con herramientas propias de la educación popular, orientadas a la construcción colectiva de conocimientos y al ejercicio del pensamiento crítico y reflexivo; el desarrollo de cada reunión se vuelca a un registro para su posterior análisis. Se han compartido no sólo los recuerdos y las emociones sino que, en relación con el lugar que la palabra escrita tiene para este grupo de alfabetizadores, también han circulado canciones y poesías.

Si bien el camino está en pleno desarrollo, ya se vislumbra fértil para los descubrimientos y la autovaloración del grupo, por ejemplo, en las siguientes expresiones:

\footnotetext{
"Nooo! ¿entonces estamos hace diez años haciendo alfabetización en los barrios?! vos sabías eso?? ¡Es un montón!!”

"uno no tiene idea dónde está... ¡Diez años! ¡Es un montón! Tenemos que celebrar"

“iinosotras tenemos que chapear ((mostrarnos)) más con todo esto ${ }^{17 ! ! "}$
}

En cuanto a la segunda estrategia prevista, tal como se señala más arriba, se trata de abordar las trayectorias educativas de los propios integrantes del grupo. Posicionados desde el enfoque conceptual y metodológico de

17 Expresiones de asombro recogidas en la reunión realizada el 30/09/2019, donde se avanzó en la reconstrucción de la memoria colectiva, a través de la elaboración conjunta de una línea de tiempo preliminar. 
las biografias educativas, se apuesta no sólo a la generación de conocimiento científico sobre las mismas, sino al impacto educativo que conlleva la elaboración de estos relatos, en lo personal y en lo colectivo.

Si bien aún no se ha avanzado en esta estrategia, se ha iniciado la ubicación de cada integrante del grupo en la línea de tiempo preliminar, mencionada más arriba. $Y$ dado que han surgido algunas expresiones "espontáneas" en torno a ello, se ha propuesto la escritura de una primera narrativa breve, acerca de cuándo, por qué y para qué iniciaron sus prácticas alfabetizadoras en la organización y cómo fue ese inicio. Los próximos pasos, se orientarán hacia la reconstrucción de las trayectorias educativas individuales (desde una perspectiva amplia de lo educativo), en espacios de producción individual y grupal, a efectos de potenciar las reflexiones y análisis colectivos como base para identificar las fuentes y las tramas de construcción colectiva de conocimiento en torno a las prácticas alfabetizadoras.

Es interesante reflexionar con Le Grand, acerca de que "[...] con frecuencia, el conocimiento es algo desencarnado, que flota en una especie de halo puramente institucional sin verdadera vinculación con las situaciones personales significativas [...]" (LE GRAND, 2004, p. 4). La reconstrucción de las biografias educativas como complementarias de la historia de vida colectiva contribuiría a que cada integrante se reencuentre como protagonista y parte activa de los procesos de aprendizaje y de construcción colectiva de conocimiento.

Como parte de la sensibilización del grupo hacia el trabajo biográfico se ha reflexionado acerca de la utilidad y el impacto que conlleva el trabajo biográfico, a partir de su propia experiencia previa en la implementación de proyectos didácticos de alfabetización (mencionados más arriba), en los cuales han trabajado con grupos de alfabetizandos en sus territorios, en el entrelazado entre reconstrucción histórica y biográfica. Los alfabetizadores señalaron que ese trabajo, además de haber sido el estímulo y la oportunidad para múltiples aprendizajes en torno a la lectoescritura, había servido también para resignificarse y revalorizarse como alfabetizandos y como militantes de una organización social, frente a un contexto socioeconómico y político hostil. Frente a esto, reafirmaron la importancia de elaborar sus propias biografias educativas: "Como que laburamos biografias con las vecinas, ipero no lo hacemos con nosotras mismas!"18.

Por último, interesa señalar que se prevé, como tercera estrategia, la integración de la memoria colectiva y biográfica, en sesiones de retroalimentación ampliadas, con la participación de los propios integrantes del grupo y de otros integrantes de las organizaciones involucradas.

Las sesiones de retroalimentación constituyen espacios de trabajo con los participantes, en donde se comparte tanto el proceso como el producto de la investigación. Se entiende a estos espacios como una instancia mínima propia de la investigación participativa, sobre el supuesto epistemológico de la posibilidad de una construcción colectiva de conocimiento a través del interjuego entre los saberes de todos los participantes, entre el conocimiento cotidiano y el conocimiento científico; constituyen espacios de aprendizaje, de educación popular con jóvenes y adultos, donde se favorece la reflexión grupal y se apuesta al fortalecimiento de los procesos participativos, a partir de la objetivación de las problemáticas de la vida cotidiana ${ }^{19}$ (SIRVENT, 2008; SIRVENT y RIGAL, 2016; LLOSA, 2000).

18 Expresión de una de las referentes de alfabetización, participante del proyecto, en una de las reuniones realizadas para la reconstrucción de la memoria colectiva, el 30/09/2019.

19 Entendemos por objetivación al proceso de construcción del conocimiento por el cual la realidad cotidiana de una población se transforma en objeto de análisis y estudio para la misma (SIRVENT, 2008). 
En el caso de esta línea de investigación, las sesiones de retroalimentación están previstas como espacios para compartir y avanzar colectivamente sobre la integración de los resultados que se hayan obtenido a través de las dos estrategias anteriores, convocando no sólo a quienes hayan participado de manera directa, sino a otros militantes de las organizaciones involucradas. Se pretende por esta vía, además, aportar a la validez de los resultados a través de la triangulación intra e intermétodo y de la validación "in situ” (como técnica no convencional de validación, propia de la IAP) (SIRVENT, 2011), con la participación de todos los involucrados.

\section{Reflexiones finales}

Se han presentado en este artículo las principales decisiones metodológicas y los primeros pasos realizados en torno a una línea de indagación actualmente en curso, en el marco de una investigación acerca de los procesos pedagógicos y psicosociales que dan cuenta de la construcción colectiva de conocimientos en experiencias desarrolladas por organizaciones y movimientos sociales. Esta línea de indagación se enfoca, como estudio de caso, en el devenir de la construcción colectiva de conocimientos acerca de las prácticas alfabetizadoras con personas jóvenes y adultas en organizaciones y movimientos sociales. Se han compartido en este artículo, los aspectos y desafios que conlleva la implementación de un abordaje colectivo y participativo para la reconstrucción de la memoria compartida y de las biografias educativas de alfabetizadores que se desempeñan en dichas organizaciones. Dado que se trata de una línea en desarrollo, está sujeta a reajustes, en el marco de un diseño flexible e interactivo (MAXWELL, 1996), propio de una estrategia metodológica general cualitativa y participativa. En parte, la elaboración de este texto también responde a la necesidad de ir registrando las decisiones y los avances realizados en la construcción del objeto en estudio, como parte de la "historia natural" de la investigación, de la observación de la propia implicación y del cuidado de la consistencia (vigilancia) epistemológica propia del quehacer científico.

Las actividades de investigación desarrolladas con los alfabetizadores referentes de estas organizaciones sociales se asientan en actividades previas de articulación de actividades de docencia, investigación y extensión universitaria, las continúan y las amplían, a través de la relación intrínseca entre la IAP y la Educación Popular en su tradición latinoamericana ${ }^{20}$. Esta relación se orienta a reforzar los aspectos participativos en la generación de conocimiento científico, entendiendo a la participación como proceso de involucramiento de aquellos sujetos tradicionalmente entendidos como "objeto de investigación", en tanto activos protagonistas en la toma de decisiones en la investigación.

Desde este posicionamiento epistemológico, político pedagógico y metodológico, se asume que, en este caso, la reconstrucción de la memoria colectiva y de las biografias educativas, conlleva y desborda su utilidad metodológica en términos de una obtención de resultados científicos, hacia otros sentidos que van más allá, en términos no sólo de la posibilidad de enriquecer la formación de alfabetizadores, sino de desarrollar colectivamente una praxis político-pedagógica en un sentido transformador frente a la realidad actual. Acercarnos a ese horizonte, parece requerir también transformar las formas de conocer.

En un contexto actual de derechización

20 El equipo de docentes investigadores forma parte de la RIAPEP: Red de Investigación Acción Participativa y Educación Popular en Universidades Públicas, en la cual se articulan equipos de universidades de Argentina y Brasil. 
política a nivel regional (BRANDAO, 2016), con la radicalización del neoconservadurismo y el neoliberalismo, ciertos rasgos parecen haber reagudizado las múltiples pobrezas: de protección, de entendimiento, de participación ${ }^{21}$. Desde diversas organizaciones sociales y comunitarias se continúa señalando la persistencia y profundización, en los territorios populares urbanos, de problemáticas estructurales en torno a las cuales trabajan (dificultades en el acceso a la tierra y la vivienda, para el acceso y la continuidad en el sistema educativo, problemas de salud y alimentación, contaminación ambiental, etc.). Asumimos la relevancia del crecimiento de las capacidades para el pensamiento reflexivo y de la construcción de conocimientos colectivos y críticos sobre las distintas problemáticas, por parte de los sujetos protagonistas, tanto universitarios como integrantes de estos grupos y movimientos, como base para el enfrentamiento de estas cuestiones y para la elaboración de alternativas superadoras y de proyectos colectivos de transformación social.

\section{Referencias}

ALHEIT, Peter. The "Biographical Question" as a Challenge to Adult Education. En: BELANGER, Paul; GEL$\mathrm{PI}$, Ettore. Lifelong Education. Países Bajos: Kluwer Academic, 1995. p. 283-298.

BARALDO, Natalia. Educación en y desde los Movimientos sociales: ¿nuevo objeto y nuevos abordajes en Educación? Algunas tendencias en estudios

21 El concepto de múltiples pobrezas es una reinterpretación de la noción misma de pobreza, sobre la base de la conceptualización integral o sistémica de las necesidades humanas. No se agota en el diagnóstico de las carencias que hacen a la satisfacción de las necesidades llamadas (tradicionalmente) básicas u obvias, sino que abarca el estudio de una compleja realidad de pobrezas (en plural) en relación con carencias en la satisfacción de necesidades fundamentales, pero no tan obvias, que se manifiestan especialmente como la pobreza de protección, la pobreza de pensamiento reflexivo o de entendimiento y la pobreza de participación (Sirvent, 2008). recientes. Cuadernos de educación, Córdoba, Universidad Nacional de Córdoba, n. 8, p. 165-176, oct. 2010. Disponible en: https://revistas.unc.edu.ar/ index.php/Cuadernos/article/view/803 Acceso en: 23/06/2017

BOURDIEU, Pierre. Cosas Dichas. España: Gedisa, 1993.

BRANDÃO, Carlos Rodrigues. Conversaciones de Carlos Brandao con María Teresa Sirvent. En: Jornadas Educación y trabajo de jóvenes y adultos a lo largo de la vida, 25-26 octubre 2016, Buenos Aires. Anais... Buenos Aires, FFyL UBA, 2016. Disponible en: https://peetiiceuba.files.wordpress.com/2017/04/jornadas2016jovenesyadultos Acceso en: 05/08/2017.

DE VILLERS, Guy. Relatos de historia de vida en formación de adultos. 1990 [S.D]. Disponible en: http://www.psicologiagrupal.cl/documentos/articulos/index.html Acceso en: 26/06/2007.

DOMINICÉ, Pierre. O que a Vida lhes Ensinou. En: NÓVOA, António; FINGER, Matthias. (Org.). 0 Método (Auto) biográfico e a Formação. Lisboa: Depto. de Recursos Humanos. Ministério da Saúde. 1988. p. 131-153.

DOMINICE, Pierre. L'histoire de vie comme processus de formation. París: L'Harmattan, 1990.

GHON, Maria da Glória. Movimientos Sociais e educação. Sao Paulo: Cortez, 1999.

GONZÁLEZ TERREROS, María Isabel; AGUILERA MORALES, Alcira; TORRES CARRILLO, Alfonso. Investigar subjetividades y formación de sujetos en y con organizaciones y movimientos sociales. En: PIEDRAHITA ECHANDÍA, Claudia; DÍAZ GÓMEZ, Álvaro; VOMMARO, Pablo (Comp.): Acercamientos metodológicos a la subjetivación política: debates latinoamericanos. Bogotá: Universidad Distrital Francisco José de Caldas: Clacso, 2013. p. 49-70.

GUELMAN, Anahí. Pedagogía y movimientos sociales: lo pedagógico y lo político en sus propuestas educativas. En HILLERT, Flora; AMEIJEIRAS, María José; GRACIANO, Nora (Ed.). La mirada pedagógica para el siglo XXI: teorías, temas y prácticas en cuestión. Buenos Aires: Editorial de la Filosofía y Letras, 2011. p. 120-130. 
JOSSO, Marie-Christine. História de vida e projeto: a história de vida como projeto e as "historias de vida" a servicio de projetos. Educação e Pesquisa, São Paulo, Universidade de São Paulo, v. 25, n. 2, p. 11-23, jul./dez. 1999. Disponible en: https://doi. org/10.1590/S1517-97021999000200002. Acceso en: 20/09/2006.

KURLAT, Marcela. El 'culto a las letras' en los procesos de alfabetización inicial de personas jóvenes y adultas. Revista Interamericana de Educación de Adultos, Pátzcuaro, México, CREFAL, Año 36, n. 1, p. 59-90, enero-junio de 2014. Disponible en: https:// www.redalyc.org/pdf/4575/457545087004.pdf . Acceso en: $20 / 02 / 2016$.

KURLAT, Marcela. Procesos de alfabetización en jóvenes y adultos: Ismael y sus laberintos de escritura. Revista Interamericana de Educación de Adultos, Pátzcuaro, México, CREFAL, Año 33, n. 2, p. 69-95, jul./ dic. 2011. Disponible en: https://www.redalyc.org/ pdf/4575/457545093005.pdf . Acceso en: 15/03/2016.

KURLAT, Marcela; LLOSA, Sandra. Fortalecimiento de procesos de alfabetización de personas jóvenes y adultas: un proyecto en marcha. En: BALMACEDA, Aníbal José... [et al.] III Encuentro Latinoamericano: Escenarios Sociales de la Educación de Jóvenes y Adultos en América Latina. Tres de Febrero: Imaginante, 2019a. p. 202-212. Disponible en: https://oei. org.ar/caeu/wp-content/uploads/2019/09/Maqueta-OEl.pdf . Acceso en: 30 oct. 2019.

KURLAT, Marcela; LLOSA, Sandra. Educación superior y movimientos sociales: articulaciones en torno a la alfabetización desde la perspectiva del derecho a la educación a lo largo de la vida. En: FELDFEBER, Myriam; ORSE, Victoria. (comp.). Educación superior como derecho: 100 años de la Reforma Universitaria. Buenos Aires: Ed. Buenos Libros. 2019b. En prensa.

LE GRAND, Jean-Louis. Puntos de referencia teóricos y éticos en historias de vida colectiva. Revista Diálogos (electrónica). n. 38, p. 7-13, 2004. Disponible en: http://www.dialogosred.net/revistas/revista38. htm . Acceso en: 15 jul. 2016.

LLOSA, Sandra.: Demandas educativas de jóvenes y adultos a lo largo de la vida: una perspectiva psicosocial. Revista Interamericana de Educación de
Adultos, Pátzcuaro, México, CREFAL, Año 39, n. 1, p. 69-90, junio 2017. Disponible en: http:/ / www.crefal. edu.mx/rieda/images/rieda-2017-1/exploraciones3. pdf Acceso en: 29/07/2017.

LLOSA, Sandra. Las biografias educativas como estrategia de investigación - formación - transformación: hallazgos y desafios. En: Congresso Internacional de Pesquisa (Auto) biográfica, 7, 2016, Cuiabá. Anais... Cuiabá, 2016. Disponible en: http://viicipa. com.br/wordpress/wp-content/uploads/2016/08/ C2T_LAS-BIOGRAFI\%CC\%81AS-EDUCATIVAS-COMO-ESTRATEGIA-DE.pdf Acceso en:28/02/2017

LLOSA, Sandra. "Será por todas estas cosas que me pasaron en la vida...": estudio de los procesos psicosociales que dan cuenta de las demandas educativas a lo largo de la vida. Revista del IICE, Buenos Aires, UBA, n. 31, p. 83-98, jul/dic 2012. Disponible en: http:// revistascientificas.filo.uba.ar/index.php/ iice/article/view/367/344 Acceso en: 12/03/2014

LLOSA, Sandra. La sesión de retroalimentación de la investigación como espacio de construcción colectiva de conocimientos: una experiencia en La Ribera de Quilmes. En: LITWIN, E. et. al. Educación, Crisis y Utopías: Análisis político y propuestas pedagógicas. Tomo I. Buenos Aires: UBA - Aique, 2000. p. 298-306.

MALDONADO, Martín. Movimientismo Estatal. Poder, Legitimidad y Territorio en 360․ Revista Cultura y representaciones sociales, México DF, Universidad Nacional Autónoma de México, v. 11, n. 21, p. 170210, set. 2016. Disponible en: http://www.scielo.org. $\mathrm{mx} / \mathrm{pdf} / \mathrm{crs} / \mathrm{v11n} 21 / 2007-8110-\mathrm{crs}-11-21-00170 . p d f$. Acceso en: 30/06/2017

MAXWELL, Joseph A. Qualitative Research Design: An interactive approach. California: Sage Publicatios, 1996.

MENGHINI, Raúl, MIGLIAVACCA, Adriana y MAS ROCHA, Stella. Enseñar en la escuela secundaria. Condiciones de trabajo en la Argentina de comienzos del siglo XXI. Poiésis, UNISUL, Santa Catarina, Brasil, v. 10, n. 17, p. 29-53, Jan/Jun 2016. http://www. portaldeperiodicos.unisul.br/index.php/Poiesis/ index. Acceso en: 20/12/2017.

MICHI, Norma; DI MATTEO, Álvaro; VILA, Diana. Movimientos populares y procesos formativos. Revista 
Polifonias, Buenos Aires, Universidad Nacional de Luján, n. 1, p. 22-41, dic. 2012. Disponible en: http:// www.polifoniasrevista.unlu.edu.ar/sites/www.polifoniasrevista.unlu.edu.ar/files/site/3\%20-\%20Michi.pdf . Acceso en: 14/04/2017

OLINDA, Ercilia; PINTO, Elismária. O círculo reflexivo biográfco na pesquisa com jovens da periferia de Maracanaú-CE. Revista @mbienteeducação. São Paulo, Universidade Cidade de São Paulo, v. 12, n. 2, p. 263-286 mai/ago 2019. Disponible en: https:// doi.org/10.26843/ae19828632v12n22019p263a286. Aceso: 4/08/2019.

PASSEGGI, Maria da Conceição. Simbolizações do percurso de escrita da narrativa autobiográfica: da experiência sensivel à experiência formadora. En: PERES, Lúcia Maria Vaz; EGGERT, Edla; KUREK, Deonir Luís. Essas coisas do imaginário: diferentes abordagens sobre narrativas (auto)formadoras. São Leopoldo: Oikos; Brasília: Liber livros, 2009. 148-161.

RHÉAUME, Jacques. El relato de vida colectivo y la aproximación clínica en ciencias sociales. Perfiles Latinoamericanos. México, Facultad Latinoamericana de Ciencias Sociales, n. 21, p. 99-116, dic. 2002. Disponible en: https://perfilesla.flacso.edu.mx/ index.php/perfilesla/article/view/295/248. Acceso en: $12 / 07 / 2019$

RIGAL, Luis. Educación, democracia y ciudadanía en la postmodernidad latinoamericana: a propósito del surgimiento de nuevos actores sociales. Revista de la Asociación de Sociología de la Educación - RASE, vol. 1, n. 3, p. 22-42, set. 2008. Disponible en: https:// ojs.uv.es/index.php/RASE/article/view/8567/8110 . Acceso en: 15/05/2012

SALTALAMACCHIA, Homero; COLÓN, Héctor; RODRíGUEZ, Javier. Historia de vida y movimientos sociales. Itzapalapa. Revista de Ciencias Sociales y Humanidades, México, UAM, n. 9, p. 321-336. Jul. 1983. Disponible en: https://revistaiztapalapa.izt.uam. $\mathrm{mx} /$ index.php/izt/article/view/847/998 . Acceso en: 13/09/2016

SAUTU, Ruth. (Comp.) El método biográfico: la reconstrucción de la sociedad a partir del testimonio de los actores. Buenos Aires: Universidad de Belgrano, 1999.
SIRVENT, María Teresa. La Educación Popular y el Nivel de Riesgo Educativo de la Población. En: Encuentro Nacional de Educación en Sectores Populares, 2, 1997, Bariloche. Mimeo.

SIRVENT, María Teresa. La Investigación Acción Participativa y la Educación Popular: su encuadre pedagógico. En: HILLERT, Flora; AMEIJEIRAS, María José; GRACIANO, Nora. (comp.): Pedagogias críticas y problemáticas socioeducativas. Buenos Aires: Facultad de Filosofía y Letras. UBA / Ed. Buenos Libros, 2011. p. 150-175.

SIRVENT, María Teresa. Educación de Adultos: Investigación, Participación, Desafios y Contradicciones. (2da ed.), Buenos Aires: Miño y Davila, 2008.

SIRVENT, María Teresa; LLOSA, Sandra. Estructura de Poder, Participación y Cultura Popular: el estudio de las demandas educativas de jóvenes y adultos, desde la perspectiva de Educación Permanente y Educación Popular. En: CASTORINA, Antonio; ORSE, Victoria. (comp.). Anuario 2011 IICE. Buenos Aires: FFyL UBA, 2012. p. 25-40.

SIRVENT, María Teresa; LLOSA, Sandra. Encuadre histórico de la Multiplicidad de Pobrezas. En: Conferencia, Concordia, Entre Ríos, 2006. AGMER (CTERA). Mimeo

SIRVENT, María Teresa; LLOSA, Sandra. Jóvenes y Adultos y educación en la Ciudad de Buenos Aires. Hacia una pedagogía de la participación. Revista del IICE, Buenos Aires, UBA, №18, p. 37-49, dic. 2001. Disponible en: http://repositorio.filo.uba.ar/handle/filodigital/5965 Acceso en: 23/03/2018

SIRVENT, María Teresa; RIGAL, Luis. Metodología de la Investigación social y educativa: diferentes caminos de producción de conocimiento (mimeos del libro en elaboración). Buenos Aires, 2016.

SIRVENT, María Teresa; LLOSA, Sandra; STEIN, Alejandra. La triangulación metodológica en investigación biográfica: aportes y desafios en el estudio de la construcción de demandas educativas a lo largo de la vida. En: ABRAHÃO, María Helena Menna Barreto; CUNHA, Jorge Luiz da; VILLAS BÔAS, Lúcia. (Org.). Pesquisa (auto)biográfica: diálogos epistêmico-metodológicos. Curitiba, Brasil: CVR, 2018. p. 107-125. 
SIRVENT, María Teresa; TOUBES, Amanda; SANTOS, Hilda; LLOSA, Sandra; LOMAGNO, Claudia. Revisión del concepto de Educación No Formal. Debates y propuestas. Revista del IICE, Buenos Aires, UBA, $\mathrm{n}$. o29, p. 41-56, marzo 2010. Disponible en: http:// repositorio.filo.uba.ar/handle/filodigital/10052 Acceso: $29 / 03 / 2019$.

SOUZA, Elizeu Clementino de. (Auto)biografias, histórias de vida e práticas de formação. En: NASCIMENTO, Antônio dias; HETKOWSKI, Tânia Maria (Org.). Memória e formação de professores. Salvador: EDUFBA, 2007. p. 59-74.

SUÁREZ, Daniel Hugo. Documentación narrativa e investigación-formación-acción en educación. En: SOUZA, Elizeu Clementino de. (Org.), (Auto)biografia e documentacao narrativa: redes de pesquisa e formação. Salvador: EDUFBA, 2015. p. 63-86.

TORRES CARRILLO, Alberto. Educación Popular y producción de conocimiento. Revista La Piragua, Panamá, CEAAL, N o 32, 8-25, 2010. Disponible en: http://ceaal.org/images/stories/LaPiragua/la\%20 piragua\%2032\%20v.pdf Acceso: 30/08/2016

VILLAGRA, Mariela; ZINGER, Sabrina; PATAGUA, Patricia. Demandas de los nuevos Movimientos Sociales en relación a la formación, el trabajo y el empleo de sus participantes y militantes: reflexiones a partir del trabajo con dos Movimientos Sociales en Jujuy. En: Jornadas de Sociología, 10, Buenos Aires. Anais... 2015. Disponible en: https: / / www.aacademica.org/000-061/879. Acceso: 12 mar. 2017.

ZIBECHI, Raúl. Autonomías y emancipaciones: América Latina en movimiento. México, D.F.: Bajo Tierra Ediciones, 2008.

Presentado en: 15.11.2019

Revisado en: 05.04.2020

Aprobado en: 07.04.2020

Sandra Mabel Llosa es Doctora en Educación por la Universidad de Buenos Aires (UBA). Profesora Asociada en el Departamento de Ciencias de la Educación - UBA. Directora de Proyectos de Investigación y de Proyectos de Extensión Universitaria en el marco del Programa Desarrollo Sociocultural y Educación Permanente: la educación de jóvenes y adultos más allá de la escuela, con sede en el Instituto de Investigaciones en Ciencias de la Educación (IICE - UBA). E-mail: sandramllosa@gmail.com 